\title{
The Helium Abundance in the Ejecta of $U$ Scorpii
}

\author{
M. P. Maxwell ${ }^{1}$, M. T. Rushton ${ }^{1}$, M. J. Darnley ${ }^{2}$, H. L. Worters ${ }^{3}$, \\ M. F. $B_{0 d e}{ }^{2}$ A. Evans ${ }^{4}$, S. P. S. Eyres ${ }^{1}$, M. B. N. Kouwenhoven ${ }^{5}$, \\ F. M. Walter ${ }^{6}$, and B. J. M. Hassall ${ }^{1}$
}

${ }^{1}$ Jeremiah Horrocks Institute, University of Central Lancashire, Preston, England email: mpmaxwell@uclan.ac.uk

${ }^{2}$ Astrophysics Research Institute, Liverpool John Moores University, Birkenhead, CH41 1LD

${ }^{3}$ South African Astronomical Observatory, PO Box 9, 7935 Observatory, South Africa

${ }^{4}$ Astrophysics Group, Keele University, Keele, Staffordshire, ST5 5BG

${ }^{5}$ Kavli Institute for Astronomy and Astrophysics, Peking University, Yi He Yuan Lu 5, Haidian Qu, Beijing 100871, China

${ }^{6}$ Department of Physics and Astronomy, Stony Brook University, Stony Brook, NY 11794-3800, USA

\begin{abstract}
U Scorpii was observed in outburst for the tenth time in January 2010. We obtained optical and near-infrared spectroscopy from which we derive a helium abundance of $N(\mathrm{He}) / N(\mathrm{H})=0.056 \pm 0.020$ from the most reliable lines available; this is lower than most other estimates and indicates that the secondary is not helium-rich, as previous studies have suggested. Velocities are found to be up to $14,000 \mathrm{~km} \mathrm{~s}^{-1}$ in broad components and up to $1,800 \mathrm{~km} \mathrm{~s}^{-1}$ in narrow line components. The reddening of $\mathrm{U}$ Sco is found to be $E(B-V)=0.14 \pm 0.12$.
\end{abstract}

Keywords. stars: novae, cataclysmic variables, abundances, individual (U Scorpii)

\section{Introduction}

U Scorpii is a recurrent nova which has been observed in outburst on 10 occasions, most recently on 2010 January $28.19 \pm 0.17$ (Schaefer et al. 2010). The system consists of a white dwarf primary with a mass close to the Chandrasekhar limit (Thoroughgood et al. 2001) and a probable subgiant secondary (Anupama \& Dewangan 2000; Hanes 1985). $\mathrm{U}$ Sco is an eclipsing system with an orbital period of 1.23 days (Schaefer \& Ringwald 1995). The system is at a distance of $12 \pm 2 \mathrm{kpc}$ and is far out of the galactic plane at a height of $4.5 \mathrm{kpc}$ (Schaefer 2010). We present optical and near-infrared spectroscopy covering the first 13 days of the 2010 outburst, which can be seen in Figures 1-3.

\section{Results}

From these spectra we determine the abundance of He I in the ejecta to be $0.012 \pm$ 0.015 from the line at $6678 \AA$. We determine the abundance of He II in the ejecta to be $0.061 \pm 0.010$ from the line at $1.163 \mu \mathrm{m}$ and $0.027 \pm 0.009$ from the line at $4686 \AA$.

We find velocities in the ejecta to be up to $14,000 \mathrm{~km} / \mathrm{s}$ in broad components and up to $1,800 \mathrm{~km} / \mathrm{s}$ in narrow components. We derive the reddening to be $\mathrm{E}(\mathrm{B}-\mathrm{V})=0.14 \pm 0.09$ from hydrogen lines on days 8.81 and 9.43 ; our result is consistent with previous studies.

The helium abundances determined here are lower than most previous estimates (Anupama \& Dewangan 2000; Evans et al. 2001); this suggests that the system does not have an over-abundance of helium and that the white dwarf is not accreting heliumrich material. Further details can be found in Maxwell et al. (2011). 


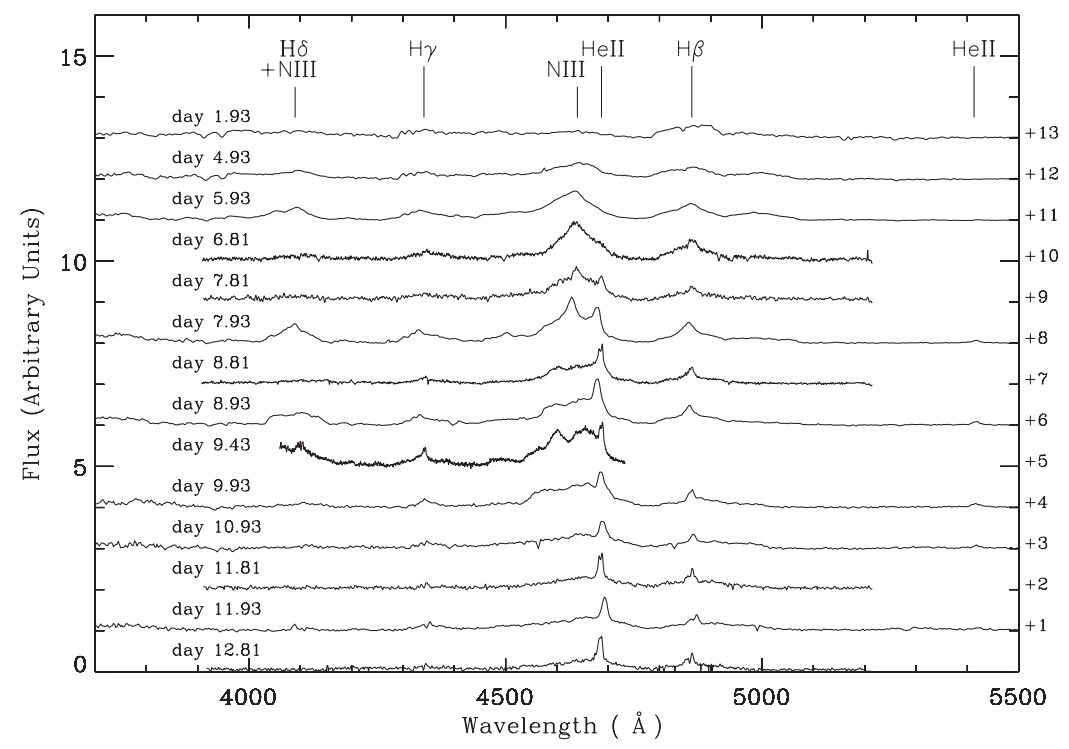

Figure 1: Spectra of U Sco from Liverpool Telescope (LT; days ending .81), Cerro Tololo Inter-American Observatory (day 9.43), and South African Astronomical Observatory (SAAO; days ending .93). Spectra are offset; flux is in arbitrary units. LT spectra are smoothed with a Gaussian profile.

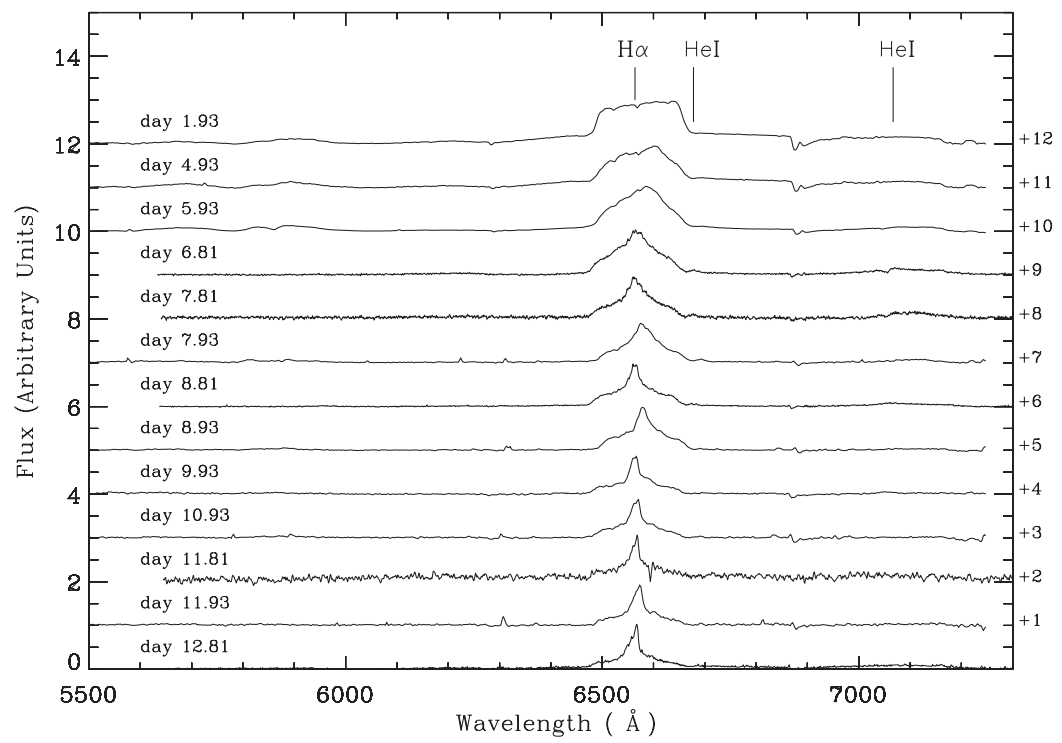

Figure 2: Spectra of U Sco taken from day 1.93 to 12.81 at LT (days ending .81) and SAAO (days ending .93). The spectra are offset as indicated and the flux is in arbitrary units. LT spectra are smoothed with a Gaussian profile. 


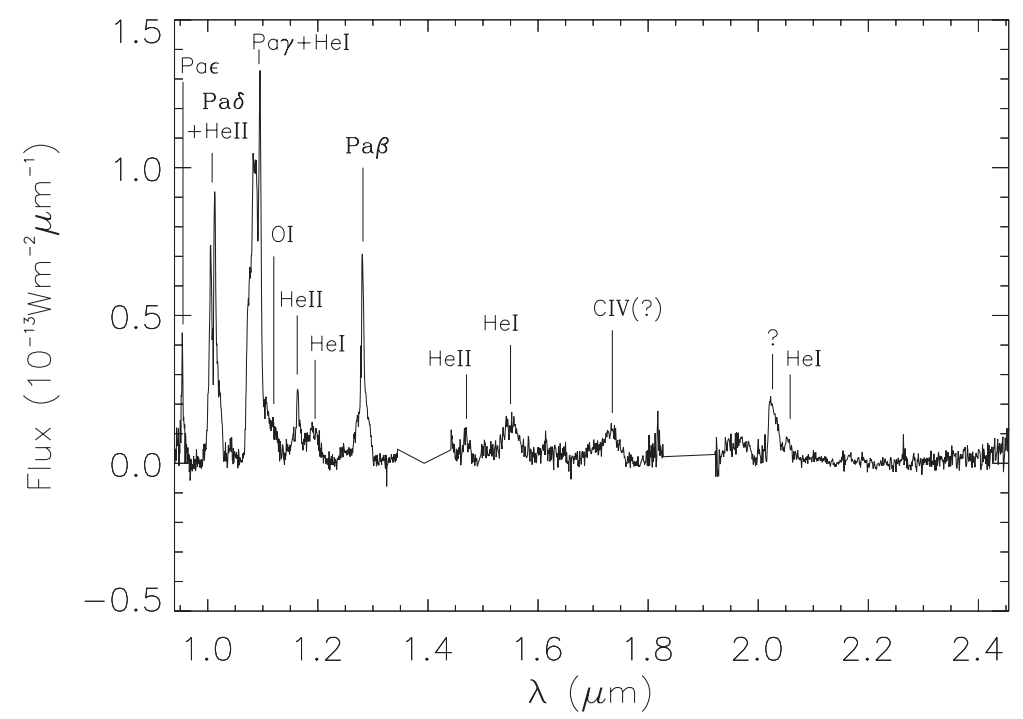

Figure 3: IR spectrum of U Sco taken at the New Technology Telescope on day 9.43 of 2010 outburst.

\section{References}

Schaefer, B. E., Harris, B. G., Dvorak, S., Templeton, M., \& Linnolt, M. 2010, IAU Circ, 9111 Thoroughgood, T. D., Dhillon, V. S., Littlefair, S. P., Marsh, T. R., \& Smith, D. A. 2001, MNRAS, 327, 1323-1333

Anupama, G. C. \& Dewangan, G. C. 2000, AJ, 119, 1359-1364

Hanes, D. A. 1985, MNRAS, 213, 443-449

Schaefer, B. E. \& Ringwald, F. A. 1995, ApJL, 447, 45+

Schaefer, B. E. 2010, ApJS, 187, 275-373

Evans, A., Krautter, J., Vanzi, L., \& Starrfield, S. 2001, A\&A, 378, 132-141

Maxwell, M. P., Rushton, M. T., Darnley, M. J., Worters, H. L., Bode, M. F., Evans, A., Eyres, S. P. S., Kouwenhoven, M. B. N., Walter, F. M., \& Hassall, B. J. M. 2011, submitted to MNRAS 